SYICHROTRON-HADIATIOA RESEARCH

J. E. Cunningham -

Oak Ridge National Laboratory*

Oak Ridge, Tennessee 37830
Cosp-821044--3

CONF $-821049--3$

DE83 001874

Synchrotron radiation is a relatively new and powerful reseerch tool that is destined to have a major impact on scientific progress in the 1980 decade. The purpose of this note, therefore, is to tell why and how this powerful probe will induce rapid scientific advance in the decade ahead.

The intensity and brightness of radiation from synchrotron source and its broad energy spectrum from the visible light through hard $x$-rays has already started a renaissance in materials science, physics, chemistry and biology. By precisely tuning synchrotron radiation to specific electron energy levels, researchers are gaining new detailed knowledge of the spatial arrangement and electronic structure of atoms in materials. This knowledge promises not only to lead to advances in theory but to aid in the developrient of materials with physical and chemical properties tailored to meet specific needs. Experinenters are using the $x$ rays of synchrotron radiation to study phase transformations as they occur, to measure how atoms are arranged relative to each other in solid solutions and liquids, to study the atomic structure of catalytic surfaces while the catalyst is in action, to observe the transient behavior of crystal growth or plastic deformation at milli-second intervals and to determine atomic arrangements and bonding distances from less than a monolayer of material, just to mention a few examples (see Physics Today, May 1981 for a more detailed discussion of these machines and the science being done). A byproduct of the unwieldy devices of medium- and high-energy physics, synchrotron radiation is rapidly becoming one of the more useful prohes of the structure of matter.

*perated by Union Carbide Corporation under contract W-7405-eng-26 with the U.S. Department of Energy

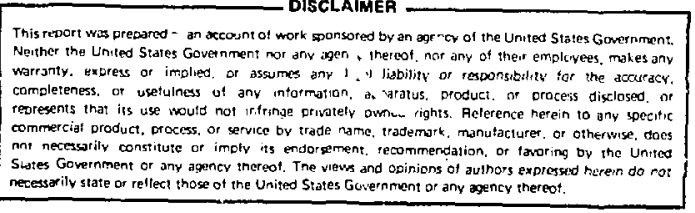

(his article, the Bublisher or recipient acknowledges publisher ar recip lont's right ty the U.S. Government's rovalty tree retain a nonexclusive, rovalty license in and to anv covering the article. 
Synchrotron Radiation Research

J. E. Cunningham

page 2

The $x$-rays from synchrotron radiation sources can give us information about electron energy levels and charge distributions as well as important characteristics about the gecmetric arrangenents of atoms and molecules in matter. These parameters determine the basic physical and chemical behavior of materials. Except in a few instances, we are unable at the present time to caiculate material properties entirely from first principles. It is very difficult to calculate even the simplest. geometric structures. Thus we must use experimental information about the geonetric structures of atoms and molecules in matter and on surfaces in advancing both our practical insights and our theoretical understanding. Property measurements of the 90-odd naturally occurring elements are relatively easy. However, most materials of technological interest consist of two or more elements in varying concentrations ard the number of conceivable combinations makes such measurements an incredible task. The goal of this new thrust is a synthesis of experimental results and theoretical calculations that will help sort out the more promising trends and lead the way to further experimentation and useful application.

\section{Advantages of Synchrotron Radiation}

Synchrotron radiaticn sources represent a dramatic improvement over conventional $x$-ray iachines, which have remained basically unchanged since 1913. The developrient of synchrotron radiation resulted in a thousandfold improvement in $x$-ray source brightness (photons/cm² sec per el fer steradian) throughout a continuous energy spectrum. With judicious use of wigglers and undulators, che level of improvement can be increased by a factor of $10^{6}$.

Of the many probes available to study matter, $x$-ray synchrotron radiation offers many advantages which have compelling appeal to researchers. Jne enticerient of synchrotron radiation is its broad and 
Synchrotron Radiation Research

J. E. Cunningham

page 3

intense energy spectrum, which is pulsed and nearly linearly polarized. The most attractive property - the unique resonances that $x$-rays have with specific electron energy levels - is not available with other probes. Synchrotron radiation provides a continuous spectrum from which we can select, with a monochromator, an energy vary near the binding energy of a bound electron. Energies are chosen to excite specific interactions involving any of the atoms throughout the periodic table. By comparison, resonances obtained with conventional electron probes are much weaker and as a consequence electron straggl ing energy-loss processes tend to dominate, and thus degrade the information and complicate interpretation of the data.

Although the use of the $x$-ray region for materials science research is still in its infancy, enough has been done to excite the imagination of researchers. Several synchrotron radiation sources are available for exploratory research in the United States. The Department of Energy operates two major facilities. One, known as the Stanford Synchrotron Radiation Laboratory, is located at the Stanford Linear Accelerator Center at Stanford University. Another, nearing completion at Brookhaven National Laboratory, is named the National Synchrotron Light Source. In addition, the National Science Foundation funds a synchrotron radiation laboratory at the University of Wisccnsin and another at Cornell University. Research proposals may be submitted by inclustry, universities, and governinent agencies to obtain beam time to conduct experiments. No charge is made for beam time unless the work is proprietary. 








Brookhaven National Laboratory. 